\title{
ESTRATÉGIA E INOVAÇÃO: análise das atividades de P\&D no setor elétrico brasileiro
}

\author{
Strategy and innovation: analysis of $\mathrm{R} \& \mathrm{D}$ activities in the Brazilian \\ electrical sector
}

\section{Carlos Olavo Quandt ${ }^{[\mathrm{a}]}$, Roberto Gregório da Silva Junior ${ }^{[\mathrm{b}]}$, Mario Procopiuck $^{[\mathrm{c}]}$}

[a] Pós-Doutorado na University of California, San Diego, professor da Pontifícia Universidade Católica do Paraná (PUCPR), Curitiba, PR - Brasil, e-mail: carlos.quandt@pucpr.br

[b] Doutorando em Administração pela Pontifícia Universidade Católica do Paraná (PUCPR), Curitiba, PR - Brasil, e-mail: roberto. gregorio@ufpr

[c] Doutorando em Administração pela Pontifícia Universidade Católica do Paraná (PUCPR), Curitiba, PR - Brasil, e-mail:mario.p@ pucpr.br

\section{Resumo}

As empresas do setor elétrico brasileiro (SEB), que atuam nas áreas de geração, transmissão e distribuição de energia elétrica, são obrigadas por lei a realizar investimentos em pesquisa e desenvolvimento (P\&D). Este trabalho analisa a inserção dos projetos oriundos desses investimentos obrigatórios na estratégia empresarial, bem como as suas implicações para a inovação no setor e seus resultados. A coleta de dados primários foi realizada por meio de questionário respondido pelos gestores de $\mathrm{P} \& \mathrm{D}$ de aproximadamente $20 \%$ das empresas do setor. Os resultados indicam que os investimentos são orientados mais ao atendimento da obrigatoriedade legal do que à produção de impactos positivos para a sociedade ou de resultados estratégicos para as organizações. Os projetos atendem a demandas internas das empresas e suas necessidades operacionais, dando pouca importância a fatores como melhoria do desempenho e geração de novos produtos, serviços ou negócios. Os resultados desta pesquisa permitem concluir que a inovação não está inserida na estratégia competitiva das empresas do setor.

Palavras-chave: Estratégia. Inovação. P\&D. Setor elétrico.

\begin{abstract}
The companies of the Brazilian electrical sector, which comprises the areas of electric power generation, transmission and distribution, are required by law to invest in RED activities. This article analyzes the strategic insertion of the projects related to those mandatory investments, as well as their implications for innovation in the sector and its results. A questionnaire was used to collect primary data from RED managers in approximately 20 percent of the companies in the sector. The findings indicate that RED investments are driven more by the
\end{abstract}


legal mandate than by the objectives of producing positive social impacts or strategic results for the organizations. The projects are geared toward internal demands and operational needs of the companies, and little importance is given to factors such as perfomance improvements or the creation of new products, services or businesses. The research findings allow us to conclude that innovation is not inserted in the competitive strategies of the sector.

Keywords: Strategy. Innovation. RED. Electrical sector.

\section{INTRODUÇÃO}

As inovações tecnológicas constituem a base do aumento da produtividade e do crescimento nos setores mais dinâmicos da economia, com efeitos econômicos e sociais muito amplos. As políticas nacionais de promoção da pesquisa e desenvolvimento (P\&D) e as estratégias corporativas refletem a importância crescente da inovação como um elemento estratégico da competitividade. Contudo, o Brasil investe pouco em P\&D. Entre os países emergentes conhecidos como BRICs (Brasil, Rússia, Índia e China), o Brasil está em último lugar em dispêndios totais em P\&D. Além disso, governo e universidades são responsáveis pela maior parte dos investimentos nas atividades de P\&D no Brasil. Enquanto em países mais desenvolvidos o investimento privado gira em torno de $70 \%$ do total dedicado à pesquisa, desenvolvimento e inovação, as empresas brasileiras respondem por menos de $40 \%$ do valor investido aqui (DE NEGRI; SALERNO, 2005).

A escassez de investimentos privados implica uma capacidade limitada de converter tecnologia em produtos e aplicações comerciais. A pesquisa industrial de inovação tecnológica (Pintec) de 2003 revelou que apenas $24 \%$ das 10 mil empresas entrevistadas faziam P\&D de forma contínua. Cerca de $12 \%$ tinham produtos diferenciados, capazes de colocá-las na liderança do mercado nacional, e menos de $2 \%$ tinham processos inovadores de impacto mundial (IBGE, 2003). Nesse contexto, é evidente a necessidade de ampliação dos investimentos em $\mathrm{P} \& \mathrm{D}$, bem como o seu alinhamento com estratégias corporativas para fortalecer a capacidade de inovação e competitividade das empresas nacionais.

Este estudo investiga a inserção estratégica dos investimentos de P\&D no setor elétrico brasileiro (SEB), que abrange empresas geradoras, transmissoras e distribuidoras de energia. O setor possui um papel vital para o país, como prestador de serviços essenciais à população e como propulsor do desenvolvimento econômico. A fiscalização e regulamentação no setor são conduzidas pela Agência Nacional de Energia Elétrica (ANEEL), criada durante a reestruturação do setor em 1996. $\mathrm{Na}$ concepção do novo modelo do SEB foi incluída a obrigatoriedade de investimentos em P\&D como forma de aumentar a eficiência do setor, "privilegiando a otimização e a produtividade como insumos, na busca da modicidade tarifária, transferindo ao consumidor final o menor preço e a máxima satisfação" (MARTINI, 2006, p. 62). Atualmente, as concessionárias são obrigadas a aplicar um percentual da sua receita operacional líquida em P\&D, correspondente a $0,2 \%$ para as distribuidoras e a $0,4 \%$ para as geradoras e transmissoras (ANEEL, 2007).

Os investimentos obrigatórios em P\&D nas áreas de geração, transmissão e distribuição já totalizam $\mathrm{R} \$ 1,15$ bilhão, aplicados em 3.695 projetos desde 1998. Tal montante de recursos ilustra a relevância potencial desses investimentos para as empresas e a sociedade em geral. Entretanto, os resultados da P\&D no setor têm sido criticados. Souza e Nicolsky (2005, p. 6) consideram que o modelo atual, apesar de, por um lado, representar um avanço significativo, "por outro lado faz inafastável a constatação de que seus resultados, no que diz respeito ao estabelecimento de uma efetiva inovação tecnológica do setor ou a um conseqüente incremento qualitativo industrial setorial, são pífios".

O presente estudo investiga estratégias utilizadas em P\&D e em inovações nas empresas pertencentes aos ramos de geração, transmissão e distribuição de energia elétrica no Brasil. Mais especificamente, o seu objetivo é analisar a inserção na estratégia empresarial dos projetos de $\mathrm{P} \& \mathrm{D}$ oriundos dos investimentos obrigatórios do setor elétrico. Inicialmente, serão trazidas à discussão algumas perspectivas estratégicas de inovação, processos de inovação tecnológica, e a inovação no ambiente regulado do setor elétrico. Na sequência, apresenta-se a 
metodologia da pesquisa, a discussão dos resultados e as considerações finais.

\section{PERSPECTIVAS ESTRATÉGICAS DA INOVAÇÃO}

Um número cada vez maior de organizações incorpora a inovação como valor fundamental de sua atuação, e mesmo como parte integrante de sua missão declarada perante o mercado e a sociedade (JOHNSTON; BATE, 2003, p. 6). As inovações podem ser vistas como o resultado da seleção, retenção e aplicação de conhecimentos para criar novos produtos, processos e características organizacionais (BUTLER, 1981, p. 764). Considerando os reflexos práticos nas atividades e resultados organizacionais, estudos mostram que o desempenho dos negócios está estreitamente relacionado com altos graus de inovação (HULL, 2004; VYAS, 2005), e que os níveis de competitividade em novos segmentos ou em novas indústrias estão vinculados à capacidade inovadora (HAGE; HOLLINGSWORTH, 2000; ADAMS; BESSANT; PHELPS, 2006, p. 21).

Embora as inovações estratégicas possam ser críticas para o sucesso (ou sobrevivência) em mercados dinâmicos, também podem se constituir em importante fonte de vantagem competitiva em mercados mais estáveis. A ação estratégica em relação à inovação vai além da simples aplicação de recursos financeiros em produtos e processos (JOHNSTON; BATE, 2003) para abranger um sequenciamento de tomada de decisões quanto à alocação de recursos, com consistência interna e alinhamento aos objetivos da organização. A coesão e a consistência na alocação de recursos normalmente são buscadas sob os ditames de arcabouços estratégicos corporativos que indicam que as decisões emanadas do sistema de gestão devem ser claramente direcionadas para a identificação e implantação de inovações em produtos e processos produtivos (ADAMS et al., 2006).

Indústrias e setores específicos inseridos em amplos sistemas de inovação são apoiados por redes de interações com outras empresas e instituições para desenvolver, adquirir e difundir novas tecnologias. Assim, criam-se processos de aprendizagem coletiva envolvendo empresas, especialmente aquelas que desenvolvem atividades de P\&D; sistemas e infraestruturas de desenvolvimento científico e tecnológico; instituições de ensino e pesquisa; e agências que atuam na transferência de tecnologias. Além do âmbito organizacional interno, as capacidades de inovação são dependentes das configurações de fatores sociais e políticos, como políticas governamentais, P\&D corporativa, sistemas de educação e treinamento, bem como a estrutura setorial específica (WARRIAN, 2004, p. 140).

No contexto tecnológico, a estratégia configura o método para o desenvolvimento e uso da tecnologia, devendo contemplar as tecnologias relevantes em uso, as trajetórias prováveis das transformações técnico-científicas, a capacitação tecnológica disponível e a seleção de alternativas para a viabilização das tecnologias necessárias ou consideradas críticas (PORTER, 1989). A estratégia tecnológica também pode compreender medidas voltadas à elevação da produtividade e da qualidade, o desenvolvimento e implementação de projetos de inovação necessários para a modernização e expansão e, ainda, incentivos para ações empreendedoras destinadas a enfrentar rupturas tecnológicas (MARCOVITCH, 1990).

A atuação em ambientes tecnologicamente dinâmicos demanda investimentos em $\mathrm{P} \& \mathrm{D}$, interna ou externamente, bem como a criação de alianças e parcerias ou a aquisição externa de tecnologias. $\mathrm{O}$ desempenho inovador está relacionado às estratégias tecnológicas adotadas pelas empresas (RIEG; ALVES FILHO, 2007). A competitividade não depende apenas das tecnologias utilizadas, mas também da eficiência com que elas são empregadas. Portanto, a competitividade depende do esforço permanente de difusão, absorção e aperfeiçoamento de tecnologias existentes, assim como do desenvolvimento de novas tecnologias (VIOT'TI, 2003).

\section{INOVAÇÃO NO SETOR ELÉTRICO}

O setor público influencia fortemente a estrutura produtiva dos setores regulados, desde aspectos como a estrutura legal e regulatória, as políticas de financiamento, a tributação e o ambiente macroeconômico, até a própria estrutura da indústria, a organização e governança corporativa e as relações entre organizações (WARRIAN, 2004, p. 140). As políticas governamentais se estendem a tentativas de desenvolver arcabouços normativos destinados a exercer controle sobre outros aspectos, como os resultados e práticas comerciais adotadas pelas empresas, e incentivos à inovação tecnológica. A 
intervenção governamental usualmente é orientada por objetivos sociais, e busca constituir sistemas de governança para direcionar os esforços das organizações públicas e privadas para alcançar esses objetivos amplos (PROCOPIUCK; FREY, 2008).

No Brasil, a gestão da P\&D no âmbito das concessionárias, segundo Martini e Maffei (2005, p. 7), é tratada sob óticas diversas. Para alguns, a obrigatoriedade dos investimentos cria possibilidades reais de inovação e aumento da competitividade. Para outros, "é uma maneira de aplicar recursos existentes para experimentar algumas idéias, sem grandes compromissos. [...] há quem considere P\&D uma obrigação a ser cumprida. Há ainda os indiferentes ao assunto".

Diante disso, as estratégias de pesquisa, desenvolvimento e inovação das empresas do SEB podem ser direcionadas por demandas diversificadas e potencialmente contraditórias. Por um lado, existe a busca de satisfação às demandas da sociedade por melhorias da qualidade no fornecimento, no uso eficiente da energia e na atenuação dos impactos ambientais. Por outro lado, há a necessidade de otimizar investimentos, aumentar a confiabilidade e reduzir os custos operacionais das empresas. $\mathrm{O}$ modelo institucional do SEB, caracterizado por um ambiente híbrido, onde coexistem empresas públicas e privadas, particulariza o tradicional conflito entre as funções econômica e social das empresas. A tensão entre os interesses corporativos e o interesse público pelos serviços por elas prestados tende a se refletirnas estratégias de investimentos obrigatórios em P\&D.

\section{METODOLOGIA}

Este estudo tem como objetivo analisar a inserção dos investimentos obrigatórios em $\mathrm{P} \& \mathrm{D}$ na estratégia competitiva das concessionárias do setor elétrico brasileiro. A pesquisa foi enfocada na análise da percepção dos gestores de P\&D dessas empresas quanto à importância relativa de diversas variáveis que expressam as relações entre investimentos, estratégias e inovação. Essa opção de delineamento da pesquisa foi adotada pela necessidade de contornar a dificuldade de obtenção de indicadores objetivos da execução da estratégia junto às concessionárias de geração, transmissão e distribuição de energia elétrica. $\mathrm{O}$ estudo foi orientado por oito questões de pesquisa:
1) Quais são as atividades de $P \& D$ com maior potencial inovador para a empresa e qual é o grau relativo de importância atribuído a essas atividades?

2) Quais são os principais fatores motivadores para a concretização dos investimentos em P\&D, em termos da sua importância relativa para a empresa nos últimos cinco anos?

Com relação à influência de variáveis na seleção e execução de projetos de P\&D:

3) Quais são as características que exercem mais influência na seleção dos projetos?

4) Qualé o grau de integração da empresa para execução dos projetos com empresas do mesmo grupo controlador e outras organizações?

5) Quais são as características mais importantes dos fornecedores para a execução dos projetos?

Quanto às expectativas em relação a resultados específicos e ao alinhamento estratégico dos projetos de P\&D:

6) Quais são as principais expectativas da empresa quanto aos resultados dos projetos?

7) Quais aspectos do alinhamento estratégico são buscados com a seleção dos projetos?

8) Quais foram os impactos efetivos dos projetos de $\mathrm{P} \& \mathrm{D}$ nos últimos cinco anos?

Para obter respostas a essas questões relacionadas às estratégias e aos resultados dos projetos de $\mathrm{P} \& \mathrm{D}$, foi realizada uma pesquisa de campo junto aos gestores de $\mathrm{P} \& \mathrm{D}$ das concessionárias de geração, transmissão e distribuição de energia elétrica. Os dados foram coletados por meio de questionário composto por 54 perguntas fechadas, contemplando as variáveis relacionadas às questões de pesquisa. A população estudada constituiu-se das 182 empresas do SEB, e a pesquisa desenvolveu-se entre agosto e outubro de 2008. A coleta de dados foi feita inicialmente por meio de questionário eletrônico disponibilizado em um 
website e, posteriormente, com o encaminhamento de questionário via e-mail. Como resultado de tais procedimentos, foi obtida uma amostra de 36 empresas, que representa aproximadamente 20\% da população estudada. A amostra foi constituída por 14 empresas do ramo de distribuição, 13 de geração e 9 de transmissão. Quanto ao controle de capital das empresas, 21 dos questionários recebidos provêm de empresas com controle privado e 15 com controle público.

Diante da constatação de que os dados analisados não se subsumem à hipótese de normalidade, procurou-se utilizar técnicas estatísticas com robustez suficiente para tolerar tal condição, especialmente para grupos superiores a 30 casos. Assim, na análise dos dados foi utilizada comparação entre grupos por meio da Analysis of Variance (ANOVA) one way, com um nível de significância de $\mathrm{p} \leq 0,05$, e estatística descritiva. Todos os cálculos foram operacionalizados por meio do software estatístico SPSS (SPSS INC., 2006).

Para avaliação da variância, antes de decidir por um teste post hoc mais adequado, foi utilizado o teste proposto por Levene. A exploração post hoc das diferenças entre as médias dos grupos, para os casos que apresentaram igualdade de variância, foi feita por meio do teste de Tukey (DMS), que, segundo Maroco (2003, p. 133), "é um dos mais robustos a desvios à normalidade e homogeneidade de variâncias". Para os casos em que não houve constatação de homogeneidade das variâncias foi lançado mão do teste de Games-Howell, que é "mais apropriado quando não há igualdade de variâncias" (SPSS INC., 2006).

\section{ANÁLISE DOS RESULTADOS}

$\mathrm{Na}$ análise dos dados, inicialmente foi verificada, através da ANOVA, a existência de diferenças nas médias das variáveis entre as empresas de geração, transmissão e distribuição. Posteriormente, com base na estatística de Levene, foram identificados os casos com e sem igualdade de variância. A seguir, para os casos de igualdade de variâncias, foi aplicado o teste post hoc de Tukey. Nos casos que apresentaram diferença de variância, foi utilizado o teste de teste post hoc de Games-Howell. A aplicação e os resultados de cada teste serão discutidos ao longo do texto, no contexto específico da análise de cada variável.
Nas seções a seguir, a discussão das questões de pesquisa será apresentada sequencialmente, a partir dos dados obtidos com as perguntas do questionário. As frequências relativas das respostas são detalhadas nas Tabelas 1 a 8, de acordo com a escala utilizada no instrumento de pesquisa para designar a percepção dos respondentes acerca do grau de importância de cada variável: $1=$ irrelevante, $2=$ pequeno, $3=$ médio, $4=$ grande, $5=$ muito grande. No texto, entre parênteses são apresentadas as médias de cada questão em relação a tal escala. Essa média é representada pelo símbolo $\mu$.

\section{Atividades com potencial de inovação}

A primeira questão de pesquisa investigou as atividades de $\mathrm{P} \& \mathrm{D}$ com maior potencial inovador e a importância atribuída pela empresa a essas atividades. Os respondentes consideraram como de "média importância" as atividades de aquisição externa da P\&D $(\mu=3,90)$, a aquisição de outros conhecimentos externos $(\mu=3,66)$, o treinamento $(\mu=3,14)$, a aquisição de máquinas equipamentos $(\mu=3,00)$ e as atividades internas de $P \& D(\mu=3,10)$, conforme detalhado na Tabela 1 . Neste conjunto de variáveis, destacou-se a importância conferida às fontes externas de $\mathrm{P} \& \mathrm{D}$ e outros conhecimentos externos, que tem a maior concentração de respostas classificando-as com importância "grande" ou "muito grande" em termos do seu potencial inovador para a empresa.

Os resultados do teste de Games-Howell indicam que o grau de importância atribuído para a atividade "projetos de engenharia e outras preparações técnicas" pelas empresas que atuam com distribuição e de transmissão são significativamente diferentes. O grupo de empresas pertencentes ao ramo de transmissão apresentou média $(\mu=3,22)$ inferior àquela do grupo do ramo de distribuição $(\mu=4,00)$. No primeiro caso, foi considerada como de média importância, enquanto no segundo atingiu o patamar de grande importância na escala utilizada.

Essa diferença pode ser explicada porque a atividade de distribuição compreende uma infraestrutura operacional mais diversificada e complexa em termos de redes, equipamentos e componentes. A Tabela 1 também indica que as atividades inovativas, em essência, são voltadas à internalização de 
TABELA 1 - Grau de importância atribuída pela empresa às atividades com potencial inovador

\begin{tabular}{lccccc}
\hline Atividades inovativas & $\mathbf{1}$ & $\mathbf{2}$ & $\mathbf{3}$ & $\mathbf{4}$ & $\mathbf{5}$ \\
\hline Aquisição externa da P\&D & - & $6 \%$ & $11 \%$ & $69 \%$ & $14 \%$ \\
Aquisição de outros conhecimentos externos & - & $6 \%$ & $34 \%$ & $49 \%$ & $11 \%$ \\
Atividades internas de P\&D & - & $29 \%$ & $31 \%$ & $29 \%$ & $11 \%$ \\
Projetos de engenharia e outras preparações técnicas & $6 \%$ & $11 \%$ & $40 \%$ & $20 \%$ & $23 \%$ \\
Treinamento & - & $15 \%$ & $62 \%$ & $15 \%$ & $9 \%$ \\
Aquisição de máquinas e equipamentos & $3 \%$ & $18 \%$ & $50 \%$ & $24 \%$ & $6 \%$ \\
\hline
\end{tabular}

tecnologias em atividades operacionais das empresas. Com essa finalidade, a inovação apresenta grau de importância situado na escala entre médio e grande, quer para o ramo de geração, transmissão ou para o de distribuição.

\section{Fatores importantes para concretização de investimentos em P\&D}

A segunda questão de pesquisa se refere aos fatores motivadores nos últimos cinco anos para decidir sobre investimentos em P\&D. Os resultados são apresentados na Tabela 2. Evidencia-se que a maior motivação é simplesmente a obrigatoriedade imposta pelas concessões e pela legislação. Essa variável é a única percebida como de grande relevância $(\mu=4,17)$. Com importância média se destacaram as melhorias nos serviços objetos da concessão $(\mu=3,24)$ e a melhoria da imagem institucional $(\mu=3,03)$. De pequena importância figuraram o aumento de desempenho econômico-financeiro $(\mu=2,31)$ e o lançamento de novos serviços ou produtos $(\mu=2,03)$. Nesse conjunto de variáveis, destaca-se a pequena importância relacionada a aspectos competitivos e de orientação ao mercado. O desenvolvimento de novos negócios $(\mu=1,93)$, a conquista de novos mercados $(\mu=1,76)$ e a redução da concorrência $(\mu=1,62)$ foram avaliados como fatores motivadores irrelevantes.

TABELA 2 - Grau de importância de diversos fatores para a concretização dos investimentos em P\&D

\begin{tabular}{|c|c|c|c|c|c|}
\hline Fatores motivadores nos últimos cinco anos & 1 & 2 & 3 & 4 & 5 \\
\hline Obrigatoriedade imposta pelas concessões e legislação & $8 \%$ & - & $8 \%$ & $36 \%$ & $47 \%$ \\
\hline Melhoria dos serviços objeto da concessão & $6 \%$ & $11 \%$ & $46 \%$ & $29 \%$ & $9 \%$ \\
\hline Aumento do desempenho econômico-financeiro & $12 \%$ & $50 \%$ & $26 \%$ & $9 \%$ & $3 \%$ \\
\hline Melhoria da imagem institucional & $8 \%$ & $25 \%$ & $28 \%$ & $33 \%$ & $6 \%$ \\
\hline Lançamento de novos produtos ou serviços & $33 \%$ & $39 \%$ & $14 \%$ & $11 \%$ & $3 \%$ \\
\hline Conquista de novos mercados & $43 \%$ & $40 \%$ & $14 \%$ & $3 \%$ & - \\
\hline Redução do nível de concorrência & $54 \%$ & $31 \%$ & $11 \%$ & $3 \%$ & - \\
\hline Desenvolvimento de novos negócios & $40 \%$ & $34 \%$ & $9 \%$ & $14 \%$ & $3 \%$ \\
\hline
\end{tabular}




\section{Fatores relacionados à seleção e execução de projetos de P\&D: características dos projetos}

Conforme apresentado na Tabela 3 , em termos de influências para seleção de projetos de P\&D, se sobressaíram com grande importância as demandas e sugestões internas da empresa $(\mu=4,17)$. Com grau médio de importância foram avaliadas as possibilidades de qualificação da equipe da empresa $(\mu=3,55)$, os custos específicos dos projetos $(\mu=3,00)$ e os retornos econômicos específicos dos projetos $(\mu=3,00)$. De pequena importância foram consideradas as possibilidades para geração de patentes, softwares e similares $(\mu=2,79)$. As proposições de fornecedores surgiram como irrelevantes $(\mu=1,86)$.

Globalmente, os dados da Tabela 3 sugerem que os esforços das empresas estão claramente mais direcionados àidentificação de projetos que permitam a incorporação de tecnologias para atendimento de suas próprias demandas. Essa orientação interna é reforçada pela importância dada a fontes externas de P\&D e outros conhecimentos externos, e pela fraca orientação às demandas e necessidades do mercado.

TABELA 3 - Características dos projetos

\begin{tabular}{lrrrrr}
\hline Características dos projetos & 1 & 2 & 3 & 4 & 5 \\
\hline Assuntos propostos por fornecedores & $54 \%$ & $17 \%$ & $17 \%$ & $11 \%$ & - \\
Demandas e sugestões internas da empresa & - & $3 \%$ & $3 \%$ & $58 \%$ & $36 \%$ \\
Custos específicos dos projetos & $3 \%$ & $11 \%$ & $69 \%$ & $14 \%$ & $3 \%$ \\
Retornos econômicos específicos dos projetos & $3 \%$ & $37 \%$ & $31 \%$ & $11 \%$ & $17 \%$ \\
Possibilidades de envolvimento da equipe da empresa & - & $21 \%$ & $18 \%$ & $44 \%$ & $18 \%$ \\
Possibilidade de qualificação da equipe da empresa & - & $17 \%$ & $23 \%$ & $43 \%$ & $17 \%$ \\
Possibilidade de gerar patentes, softwares ou similares & $17 \%$ & $29 \%$ & $23 \%$ & $23 \%$ & $9 \%$ \\
\hline
\end{tabular}

\section{Fatores relacionados à seleção e execução de projetos de P\&D: integração com outras organizações para execução de P\&D}

Considerando o grau de integração das empresas na execução de atividades de $\mathrm{P} \& \mathrm{D}$, as universidades assumiram média importância $(\mu=3,83)$. Com pequena importância foram avaliados os centros de P\&D $(\mu=2,83)$, as outras empresas do setor elétrico $(\mu=2,66)$ e as empresas de consultoria e similares $(\mu=2,21)$. Os fabricantes e fornecedores do setor elétrico obtiveram posição de irrelevância na avaliação $(\mu=1,79)$. Observe-se a Tabela 4.

O teste post hoc de Tukey aponta que as atividades de P\&D e outras atividades de inovação possuem diferentes graus de integração em "empresas de um mesmo controlador" nos ramos de distribuição e transmissão. No ramo de distribuição essa integração apresenta pequeno grau de importância $(\mu=2,64)$, ao passo que no ramo de transmissão é de grande importância $(\mu=4,22)$ a execução das atividades vinculadas à inovação dentro de um mesmo grupo controlador.

Globalmente, os resultados indicam aproximação das empresas com universidades e centros de P\&D, e com as demais empresas do setor. Surgem indícios de que a fonte de inovação no SEB pode estar mais vinculada à pesquisa básica que a tentativas de buscar soluções oriundas de fornecedores e empresas de consultoria. 
QUANDT, C. O.; SILVA Jr., R. G.; PROCOPIUCK, M.

TABELA 4 - Grau de integração da empresa para execução da P\&D

\begin{tabular}{lrrrrr}
\hline Entidades & $\mathbf{1}$ & $\mathbf{2}$ & $\mathbf{3}$ & $\mathbf{4}$ & $\mathbf{5}$ \\
\hline Empresas do mesmo grupo controlador & $21 \%$ & $12 \%$ & $12 \%$ & $26 \%$ & $29 \%$ \\
Empresas do setor elétrico (fora do grupo controlador) & $6 \%$ & $33 \%$ & $50 \%$ & $11 \%$ & - \\
Universidades públicas e provadas & - & $6 \%$ & $25 \%$ & $44 \%$ & $25 \%$ \\
Fabricantes e fornecedores do setor elétrico & $51 \%$ & $23 \%$ & $20 \%$ & $3 \%$ & $3 \%$ \\
Centros de P\&D & $14 \%$ & $17 \%$ & $46 \%$ & $11 \%$ & $11 \%$ \\
Empresas de concultoria e similares & $20 \%$ & $51 \%$ & $20 \%$ & $6 \%$ & $3 \%$ \\
\hline
\end{tabular}

\section{Fatores relacionados à seleção e execução de projetos de P\&D: características dos fornecedores}

$\mathrm{Na}$ seleção de projetos, quanto às características dos fornecedores, foi atribuída grande importância para o nível de qualificação e experiência da equipe $(\mu=4,17)$. Com grau médio de importância despontaram a credibilidade $(\mu=3,97)$ e as instalações e laboratórios $(\mu=3,52)$. Com pequena importância foram considerados os relacionamentos anteriores com o fornecedor $(\mu=2,93)$ e a localização do fornecedor na mesma região de atuação da empresa $(\mu=2,86)$. A Tabela 5 dispõe os dados assim:

TABELA 5 - Características dos fornecedores

\begin{tabular}{|c|c|c|c|c|c|}
\hline Características dos fornecedores & 1 & 2 & 3 & 4 & 5 \\
\hline Fornecedor com sede na região de atuação da empresa & $31 \%$ & $9 \%$ & $14 \%$ & $37 \%$ & $9 \%$ \\
\hline Relacionamento anterior com o fornecedor & $23 \%$ & $11 \%$ & $23 \%$ & $37 \%$ & $6 \%$ \\
\hline Credibilidade do fornecedor & $14 \%$ & - & $3 \%$ & $43 \%$ & $40 \%$ \\
\hline Nível de qualificação e experiência da equipe do fornecedor & $14 \%$ & - & - & $29 \%$ & $57 \%$ \\
\hline Laboratórios e instalações do fornecedor & $14 \%$ & $3 \%$ & $31 \%$ & $14 \%$ & $37 \%$ \\
\hline
\end{tabular}

Os resultados da Tabela 4 colocaram os fornecedores como irrelevantes quanto à sua "integração com fabricantes do setor elétrico", e os da Tabela 3 qualificaram como irrelevantes "os assuntos propostos por fornecedores", quando se trata de busca de novas alternativas para inovação. $\mathrm{Na}$ Tabela 5 , que trata da qualificação dos fornecedores como executantes de projetos, a ênfase é atribuída à infraestrutura e para a credibilidade que eles detêm, e para a capacitação técnica que podem oferecer ou transmitir para as empresas do SEB.

\section{Expectativas e alinhamento estratégico dos projetos de $\mathrm{P} \& \mathrm{D}$ : expectativas da empresa}

Em relação às expectativas das empresas na seleção de projetos de $\mathrm{P} \& \mathrm{D}$, conforme apresentado na Tabela 6 , importância média foi atribuída à redução de custos operacionais $(\mu=3,07)$. Foi dada pequena importância à atenuação dos impactos ambientais $(\mu=2,76)$, ao aumento dos lucros $(\mu=2,59)$, aumento do faturamento $(\mu=2,41)$, desenvolvimento de 
TABELA 6 - Expectativas da empresa

\begin{tabular}{lrrrrr}
\hline Expectativas da empresa & $\mathbf{1}$ & $\mathbf{2}$ & $\mathbf{3}$ & $\mathbf{4}$ & $\mathbf{5}$ \\
\hline Aumento do faturamento & $14 \%$ & $49 \%$ & $20 \%$ & $11 \%$ & $6 \%$ \\
Aumento do lucro & $14 \%$ & $46 \%$ & $17 \%$ & $9 \%$ & $14 \%$ \\
Redução de custos operacionais & $9 \%$ & $31 \%$ & $11 \%$ & $29 \%$ & $20 \%$ \\
Melhoria da economia local/regional & $29 \%$ & $23 \%$ & $23 \%$ & $20 \%$ & $6 \%$ \\
Melhoria dos indicadores sociais locais/regionais & $41 \%$ & $11 \%$ & $33 \%$ & $15 \%$ & - \\
Atenuação de impactos ambientais & $11 \%$ & $31 \%$ & $26 \%$ & $17 \%$ & $14 \%$ \\
Melhoria nos serviços para consumidores & $17 \%$ & $17 \%$ & $9 \%$ & $37 \%$ & $20 \%$ \\
Desenvolvimento de novos negócios & $14 \%$ & $43 \%$ & $23 \%$ & $20 \%$ & - \\
Lançamento de novos produtos/serviços & $31 \%$ & $34 \%$ & $14 \%$ & $14 \%$ & $6 \%$ \\
\hline
\end{tabular}

novos negócios $(\mu=2,41)$, melhoria da economia local e regional $(\mu=2,38)$ e ao lançamento de novos produtos e serviços $(\mu=2,31)$.

Tendo em conta o grau de influência na seleção de projetos de $\mathrm{P} \& \mathrm{D}$ no que diz respeito à preocupação com a "melhoria dos indicadores sociais em âmbito local ou regional", o teste post hoc de Tukey indica diferenças significativas entre os ramos de distribuição e transmissão. No ramo de transmissão a influência é irrelevante $(\mu=1,67)$, mas no ramo de distribuição a melhoria dos indicadores sociais locais ou regionais tem grau médio de importância $(\mu=3,29)$.

Quanto às expectativas das empresas em relação à $\mathrm{P} \& \mathrm{D}$ para melhoria nos serviços para os consumidores, o teste de Games-Howell indica uma diferença significativa entre os ramos de distribuição e de transmissão. A média do ramo de distribuição $(\mu=4,21)$ foi superior à do ramo de transmissão $(\mu=3,96)$.

Em consonância com os resultados obtidos na Tabela 1, os dados da Tabela 6 reforçam que o foco da P\&D está voltado essencialmente para melhorias de processos internos e tem como objetivo principal a redução de custos. Como a Tabela 6 trata de expectativas que as empresas detêm quando da seleção de projetos de $\mathrm{P} \& \mathrm{D}$, talvez aqui sejam encontrados alguns indícios de respostas para a pequena relevância dos projetos executados em termos do seu impacto social ou de resultados estratégicos para as próprias empresas.

\section{Expectativas e alinhamento estratégico dos projetos de P\&D: alinhamento estratégico da P\&D}

No tocante à aderência do alinhamento estratégico visado com a seleção de projetos de $\mathrm{P} \& \mathrm{D}$, conforme detalhado na Tabela 7, foi considerada grande a influência das necessidades operacionais $(\mu=4,03)$, e média em relação às diretrizes estratégicas da empresa $(\mu=3,59)$. Sobressaiu-se como de pequena relevância a aderência dos projetos às políticas governamentais $(\mu=2,34)$.

Na questão relativa à influência da "melhoria nos serviços para os consumidores" na seleção de projetos de P\&D, o teste de Tukey indicou que houve diferença significativa entre os ramos de distribuição e de transmissão. A média do ramo de distribuição $(\mu=4,21)$ foi superior à do ramo de transmissão $(\mu=3,96)$. Nesses dois ramos, considerando o erro padrão da média $(0,32)$, o grau de importância da variável em questão encontra-se entre médio e grande. Essas constatações, envolvendo a geração, transmissão e distribuição, reforçam as diferenças constatadas entre os ramos no que se refere à proximidade com os consumidores, ou seja, com os clientes finais dos serviços de energia elétrica. As geradoras produzem a energia que é entregue às transmissoras, que, por sua vez, repassam-na às distribuidoras. Essas últimas é que atuam diretamente com os consumidores.

Os resultados obtidos para as questões da Tabela 7 expressam o comportamento típico de 
TABELA 7 - Alinhamento estratégico

\begin{tabular}{lrrrrr}
\hline Alinhamento estratégico & $\mathbf{1}$ & $\mathbf{2}$ & $\mathbf{3}$ & $\mathbf{4}$ & $\mathbf{5}$ \\
\hline Aderência às diretrizes estratégicas da empresa & $3 \%$ & $11 \%$ & $25 \%$ & $31 \%$ & $31 \%$ \\
Aderência às necessidades operacionais da empresa & - & $9 \%$ & $11 \%$ & $40 \%$ & $40 \%$ \\
Aderência às políticas governamentais & $23 \%$ & $26 \%$ & $29 \%$ & $23 \%$ & - \\
Aderência às necessidades dos consumidores & $6 \%$ & $26 \%$ & $23 \%$ & $31 \%$ & $14 \%$ \\
\hline
\end{tabular}

indicadores de empresas atuantes em um mercado fortemente monopolizado e com ambiente regulado, como é o caso das empresas do SEB. Até mesmo a busca de lucro não influencia de forma significativa a seleção de projetos de P\&D. Assim, os resultados também podem ser indicadores da falta de inserção da inovação na estratégia competitiva das empresas do setor.

\section{Impacto da P\&D nos resultados dos últimos cinco anos}

A última questão de pesquisa avaliou os impactos efetivos dos projetos de $\mathrm{P} \& \mathrm{D}$ nos últimos cinco anos. Conforme observado anteriormente, a análise está baseada na percepção dos próprios gestores de $\mathrm{P} \& \mathrm{D}$ quanto aos impactos observados, diante da impossibilidade de obtenção de dados quantitativos que possam ser diretamente correlacionados aos investimentos de cada empresa. A despeito dessa limitação, os resultados da pesquisa permitem identificar a importância relativa dos impactos dos investimentos nas diversas categorias de indicadores de desempenho.

Ao expressar, com base nos últimos cinco anos, o impacto dos resultados de P\&D sobre o desempenho das empresas, as médias obtidas apontam para a irrelevância de estratégias dirigidas para a atenuação de impactos ambientas $(\mu=1,97)$, a melhoria dos indicadores locais e regionais $(\mu=1,90)$, o aumento do faturamento $(\mu=1,72) \mathrm{e}$ do lucro $(\mu=1,69)$, a melhoria da economia local/ regional $(\mu=1,69)$ e para o lançamento de novos produtos $(\mu=1,66)$. Atingiu nível de pequena importância a redução dos custos operacionais $(\mu=2,10)$. Os dados são assim apresentados na Tabela 8:

TABELA 8 - Grau de impacto dos resultados da P\&D nos últimos cinco anos

\begin{tabular}{lccccc}
\hline Variáveis de desempenho & $\mathbf{1}$ & $\mathbf{2}$ & $\mathbf{3}$ & $\mathbf{4}$ & $\mathbf{5}$ \\
\hline Aumento do faturamento & $48 \%$ & $39 \%$ & $12 \%$ & - & - \\
Aumento do lucro & $52 \%$ & $39 \%$ & $6 \%$ & $3 \%$ & - \\
Redução de custos operacionais & $21 \%$ & $50 \%$ & $18 \%$ & $12 \%$ & - \\
Melhoria da economia local/regional & $50 \%$ & $31 \%$ & $19 \%$ & - & - \\
Melhoria dos indicadores sociais locais/regionais & $41 \%$ & $32 \%$ & $24 \%$ & - & $3 \%$ \\
Atenuação de impactos ambientais & $29 \%$ & $50 \%$ & $12 \%$ & $9 \%$ & $0 \%$ \\
Melhoria nos serviços para os consumidores & $26 \%$ & $29 \%$ & $29 \%$ & $12 \%$ & $3 \%$ \\
Desenvolvimento de novos negócios & $47 \%$ & $24 \%$ & $29 \%$ & - & - \\
Lançamento de novos produtos/serviços & $44 \%$ & $53 \%$ & $3 \%$ & - & - \\
\hline
\end{tabular}


O teste de Tukey indicou que há diferença significativa entre as empresas geradoras e distribuidoras no que se refere aos impactos da P\&D na "melhoria dos serviços para consumidores". Nesse quesito também foi observado que a média do ramo de geração $(\mu=1,64)$, situada no patamar de irrelevância, ficou bem abaixo da média do ramo de distribuição $(\mu=3,00)$, enquadrado no grau médio na escala. Essa situação talvez seja motivada pelo fato de que as distribuidoras atuam mais próximas aos consumidores, como já mencionado na análise dos resultados da Tabela 7 .

$\mathrm{Na}$ avaliação do impacto dos resultados de P\&D no "desenvolvimento de novos negócios", o teste de Tukey indicou que houve diferenças significativas entre os ramos de distribuição e transmissão. No ramo de distribuição $(\mu=1,71)$, a importância do P\&D para desenvolver novos negócios é irrelevante, e no ramo de transmissão $(\mu=2,67)$ é pequeno. Também ocorreu diferença significativa entre os ramos de transmissão e geração. O ramo de transmissão apresentou média bem inferior àquela do ramo de geração $(\mu=1,27)$. No ramo de geração, a $\mathrm{P} \& \mathrm{D}$ como fonte de novos negócios figurou como irrelevante. Note-se que, embora haja diferenciação quanto à utilização da P\&D como meio de desenvolvimento de novos negócios, essa estratégia, em termos de importância, chega tão somente a patamares médios.

De modo geral, os resultados da Tabela 8 demonstram que o potencial da P\&D para melhoria dos resultados das empresas tem sido pequeno. A percepção do impacto da $\mathrm{P} \& \mathrm{D}$ quanto à produção de externalidades positivas no seu ambiente externo é ainda menor. Um dos melhores índices foi obtido pela potencialidade de redução de custos operacionais $(\mu=2,10)$. Esse dado parece estar estreitamente relacionado aos resultados das atividades inovativas vinculadas ao aperfeiçoamento dos processos produtivos internos, avaliadas na Tabela 1 e corroborados pelos resultados expressos na Tabela 6 .

\section{CONSIDERAÇÕES FINAIS}

De forma geral, as evidências da pesquisa indicam que as atividades de inovação não apresentam grande importância para estratégias de ampliação do nível de competitividade ou do potencial para geração de novos negócios entre as empresas do setor. Os impactos das inovações não chegam a ultrapassar o âmbito de melhorias em processos internos das empresas. Os resultados estão fracamente vinculados ao desempenho global da empresa e às melhorias de indicadores ambientais e sociais. A utilização das inovações para elevação da lucratividade de empresas do SEB tem potencial estratégico considerado bastante reduzido.

A seleção de projetos de $\mathrm{P} \& \mathrm{D}$ procura suprir demandas internas das empresas e são voltados para atender às suas necessidades operacionais. Fatores como melhoria do desempenho econômicofinanceiro e geração de novos negócios, de produtos ou de serviços foram considerados de pequena importância. Além disso, a forte orientação dos investimentos para o atendimento de demandas e problemas operacionais pode estar comprometendo um maior grau de interações com fornecedores e fabricantes do setor.

Nesse ponto, destaca-se que o core business das concessionárias não está diretamente relacionado com a produção e comercialização de produtos, serviços, softwares e outros eventuais resultados da P\&D. Nessas empresas, em geral, ainda existem limitações para a adoção de medidas de proteção e exploração comercial das soluções tecnológicas oriundas dos projetos de $\mathrm{P} \& \mathrm{D}$. A irrelevância das interações e propostas de P\&D vinculadas aos fornecedores e fabricantes do setor é reveladora nesse sentido.

Destaca-se também que as políticas governamentais procuram estimular as externalidades positivas e reduzir os impactos negativos decorrentes das atividades das empresas dos setores regulados. As inovações no setor, em tese, teriam a capacidade de minimizar impactos ambientais causados pelas suas atividades e, ao mesmo tempo, ampliar a agregação de valores nos serviços prestados à sociedade. Com a realização desses objetivos, as inovações teriam um papel importante no desenvolvimento de economias locais e regionais. Entretanto, diferente de tais intenções, a pesquisa indica que os resultados dos investimentos em P\&D pouco têm contribuído para o desenvolvimento local e regional e, além disso, tais objetivos ainda têm pouca relevância para as empresas que atuam no SEB.

Os resultados da pesquisa não indicam o alinhamento dos esforços deinovação com as estratégias corporativas. De modo geral, constatou-se o contrário. Os gestores de $\mathrm{P} \& \mathrm{D}$ atribuem média importância aos diversos tipos de atividades inovativas vinculadas 
ao desempenho da organização, ou no que se refere aos seus reflexos em variáveis importantes para o ambiente em que se inserem. Mesmo considerando as expectativas de retorno para as empresas e o fato de que as atividades de inovação tendem a produzir resultados de longo prazo, os resultados obtidos até o momento indicam que o retorno social desses investimentos é pouco promissor. Especificamente no ramo de distribuição de energia elétrica, já foram encontrados indícios de que os investimentos em $\mathrm{P} \& \mathrm{D}$ não estariam apresentando impactos significativos na qualidade dos serviços nesse segmento (SILVA Jr., 2008).

Os resultados suscitam indagações críticas quanto aos motivos pelos quais a $\mathrm{P} \& \mathrm{D}$ não tem obtido resultados estrategicamente importantes, diante dos volumes consideráveis de investimentos realizados no período. As respostas a essa questão transcendem o escopo desta pesquisa, e talvez possam ser encontradas em análises mais específicas do perfil dos projetos implementados. Aqui surgem duas possibilidades: a) se os projetos têm prazo de retorno maior que o horizonte de cinco anos contemplado aqui, seria prematuro julgar os resultados obtidos; b) se, entretanto, os projetos deveriam produzir contribuições significativas ao longo desse período, abrem-se importantes possibilidades para se investigar a própria concepção e execução dos projetos, ou os critérios de avaliação utilizados que levaram às conclusões expressas nas respostas à pesquisa. $\mathrm{A}$ análise das intenções futuras das empresas em relação aos projetos de $\mathrm{P} \& \mathrm{D}$ propicia alguns indícios de respostas para a pequena relevância dos projetos executados, principalmente quanto à produção de impactos positivos para a sociedade ou de resultados estratégicos para as próprias empresas.

Os resultados da pesquisa indicam que o fator mais importante para a concretização dos investimentos em P\&D no SEB tem sido a obrigatoriedade legal imposta pelas concessões elegislação. Entretanto, é preocupante constatar que a inovação não está inserida na estratégia competitiva das empresas, apesar do volume significativo de investimentos em P\&D e das intenções subjacentes às diretrizes estratégicas e políticas para desenvolvimento do SEB. Os resultados indicam que os investimentos estão sendo realizados mais em virtude da obrigatoriedade legal imposta do que pelos seus potenciais de elevação do desempenho das empresas e de desenvolvimento local e regional.
Os resultados também levam à recomendação de que sejam realizados novos estudos sobre a concepção, seleção e execução dos projetos de $\mathrm{P} \& \mathrm{D}$, bem como sobre alternativas para que os investimentos obrigatórios em P\&D ampliem sua capacidade de contribuir para retornos sociais. Nesse aspecto, um referencial importante para a reflexão sobre as políticas de inovação no SEB é a constituição de sistemas de governança que integram estratégias e instituições orientadas à ação adaptativa e antecipativa em políticas de inovação. Sob essa perspectiva, enfatiza-se o desenvolvimento de condições sociopolíticas que permitem que as definições das trajetórias tecnológicas de longo prazo integrem objetivos estratégicos de competitividade e desenvolvimento econômico e social, contemplando as necessidades das diversas partes interessadas, no âmbito público e privado.

\section{REFERÊNCIAS}

ADAMS, R.; BESSANT, J.; PHELPS, R. Innovation management measurement: a review. International Journal of Management Reviews, New York, v. 8, n. 1, p. 21-47, 2006.

AGÊNCIA NACIONAL DE ENERGIA ELÉTRICA ANEEL. Investimentos em P\&D: inovação tecnológica a serviço do desenvolvimento sustentável. Revista Pesquisa e Desenvolvimento, São Paulo, ANEEL, v. 2, p. 8-9, 2007.

BUTLER, R. J. Innovations in organizations: appropriateness of perspectives from small group studies for strategy formulation. Human Relations, New York, v. 34, n. 9, p. 763-788, 1981.

DE NEGRI, A.; SALERNO, M. (Org.). Inovações, padrões tecnológicos e desempenho das firmas industriais brasileiras. 2. ed. Brasília: IPEA, 2005.

HAGE, J.; HOLLINGSWORTH, J. R. A strategy for the analysis of idea innovation networks and institutions. Organization Studies, New York, v. 21, n. 5, p. 971 1004, 2000.

HULL, F. M. Innovation strategy and the impact of a composite model of service product development on performance. Journal of Service Research, New York, v. 7, n. 2. p. 167-180, 2004. 
INSTITUTO BRASILEIRO DE GEOGRAFIA E ESTATÍSTICA - IBGE. Pesquisa Industrial de Inovação Tecnológica - PINTEC 2003. Disponível em: $<$ http://www.ibge.gov.br/home/estatistica/economia/ industria/pintec/2003 >. Acesso em: 1 out. 2008.

JOHNSTON Jr., R. E.; BATE, J. D. The power of strategy innovation: a new way of linking creativity and strategic planning to discover great business opportunities. New York, NY, USA: American Management Association, 2003.

MARCOVITCH, J. Tecnologia e competitividade. In: CICLO MODULAR DO PROTAP. Módulo II -Sessão C\&T e a geopolítica mundial. São Paulo: USP/FEA/ IA, 1990.

MAROCO, J. Análise estatística: com utilização do SPSS. 2. ed. Lisboa, Portugal: Sílabo, 2003.

MARTINI, J. S. C.; MAFFEI, O. M. A efetividade dos ciclos de pesquisa e desenvolvimento em empresas de energia elétrica. In: SEMINÁRIO NACIONAL DE PRODUÇÃO E TRANSMISSÃO DE ENERGIA ELÉTRICA, 18., 2005, Curitiba. Anais... Curitiba: SNPTEE, 2005.

MARTINI, S. Eficiência em busca da modacidade tarifária. Revista P\&D, Brasília, 2006. Disponível em: <http:// www.aneel.gov.br/arquivos/PDF/Revista_PD.pdf $>$. Acesso em: 1 out. 2008.

PORTER, M. E. Vantagem competitiva: criando e sustentando um desempenho superior. Rio de Janeiro: Campus, 1989.

PROCOPIUCK, M.; FREY, K. Governança e redes em políticas de caráter público. In: ENCONTRO DE ADMINISTRAÇÃO PÚBLICA E GOVERNANÇA, 3., 2008. Salvador. Anais... Salvador, BA: ANPAD, 2008.

RIEG, D. L.; ALVES FILHO, A. G. Estratégias tecnológicas e desempenhos inovadores das PMEs de equipamentos médico-hospitalares de São Carlos e Ribeirão Preto. Produção, v. 17, n. 2, p. 273-285, 2007.

SILVA Jr., R. G. Pesquisa e desenvolvimento e a qualidade no fornecimento de energia elétrica. In: SIMPÓSIO DE GESTÃO DA INOVAÇÃO TECNOLÓGICA, 25., 2008, Brasília. Anais... Brasília: Associação Nacional de Pós-Graduação e Pesquisa em Administração, 2008.
SOUZA, F. L. A.; NICOLSKY, R. Uma alternativa para a consolidação e institucionalização do P\&D. In: SEMINÁRIO NACIONAL DE PRODUÇÃO E TRANSMISSÃODEENERGIA ELÉTRICA, 18., 2005, Curitiba. Anais... Curitiba: SNPTEE, 2005.

STATISTICAL PACKAGE FOR THE SOCIAL SCIENCES - SPSS. V. 15.0. Chicago, Illinois: SPSS Inc., 2006.

VIOTTI, E. B. Fundamentos e evolução dos indicadores de CT\&I. In: VIOTTI, E. B.; MACEDO, M. M. (Org.). Indicadores de ciência, tecnologia e inovação. Campinas: Unicamp, 2003. p. 41-88.

VYAS, V. Imitation, incremental innovation and climb down: a strategy for survival and growth of new ventures. Journal of Entrepreneurship, v. 14, n. 2, p. 103-116, 2005.

WARRIAN, P. Measuring up in steel: performance measurement and innovation policy in the canadian steel industry. International Review of Administrative Sciences, v. 70, n. 1. p. 137-155, 2004.

Recebido: 05/06/2009

Received: 06/05/2009

Aprovado: 10/10/2009

Approved: 10/10/2009 